(C) 1981. The Genetical Society of Great Britain

\title{
THE POPULATIONAL EFFECTS OF INBREEDING IN TRIBOLIUM
}

\author{
DAVID E. McCAULEY* and MICHAEL J. WADE \\ Department of Biology, The University of Chicago, Chicago, Illinois 60637
}

Received 11. viii.80

\section{SUMMARY}

Replicated populations of the flour beetle, Tribolium castaneum, were reared under nine population structures consisting of combinations of three effective population sizes and three interdemic migration rates. For each treatment, the expected loss of genetic variance within demes owing to the cumulative effects of finite population size was calculated and related to observed changes in density-dependent population growth rates. A strong positive correlation was demonstrated between the predicted decline in within-deme genetic variar ${ }_{1} c e$ and the degree to which a deme's rate of increase declined over time. Notably, changes in the rate of population increase could be detected when the predicted loss in genetic variance was as low as 10 per cent.

\section{INTRODUCTION}

This is a study of the cumulative effects of finite population size on the loss of within-population genetic variance and the effects of this loss of variance on the density-dependent growth of populations of the flour beetle, Tribolium castaneum. The theoretical rate of decay of genetic variance within finite populations owing to inbreeding and genetic drift has been well studied (Crow and Kimura, 1970). Given a population's history of the numbers of breeding adults each generation and the mating structure, one can use established theory to predict expected changes in a population's level of heterozygosity. We have manipulated the numbers of breeding adults and the migration rates in experimental populations of $T$. castaneum and observed the response of the ecological character, population growth rate, to these manipulations. The effects of loss of heterozygosity have been previously studied for many phenotypic characteristics of individuals in a variety of organisms with the general finding that "fitness characters" (i.e., fecundity, viability, developmental period) are most affected (Wright, 1977). Population growth rate, however, is a characteristic of the population as a whole, and is determined in a complex way by the ecological interactions of its component individuals.

Some of these interactions in Tribolium have been well studied, such as the cannibalistic interactions which are known to vary with the age, sex, genotype, and density of both cannibal and victim (Sonleitner, 1961; King and Dawson, 1972; Sokoloff, 1974; 1977). Other interactions, such as male-male reproductive interference (Birch et al., 1951; Osborne, 1968), release of toxic quinones (Park, 1934; 1935; 1938a; Park and Woollcott, 1937), and larval-larval jostling (Park, 1938b; Mertz and Robertson, 1970:

* Present Address: Department of Biology, Vanderbilt University, Nashville, Tennessee 37235 
Kence, 1973; McCauley, 1978), are less well understood but are known to vary between genetic strains. Because of the density-dependent nature of these interactions between individuals, it is not clear how the loss of genetic variance within a population would affect its rate of growth. For example, in a Tribolium population whose numbers are regulated in a density-dependent fashion, a reduction in fecundity would lower the rate of densitydependent cannibalistic interactions such that a highly fecund and highly cannibalistic population may grow at approximately the same rate as a less fecund and less cannibalistic population.

Population growth in this experiment is defined as the numbers of $F_{1}$ adults produced by a given number of randomly mating parental adults in a fixed period of time in a fixed amount of resource. As a result of those mechanisms of population growth and regulation mentioned above (see King and Dawson, 1972; Mertz, 1972 and Sokoloff, 1974 for reviews) population growth rate is a complex function of the density-dependent interactions of individuals in addition to individual traits such as fecundity and development time. Several studies (McCauley, 1978; Wade, 1979; McCauley and Wade, 1980) have shown that large observed differences in population growth rate between strains cannot be predicted from studies of the demographic characteristics of single individuals removed from their ecological context. In one case, McCauley and Wade (1980) found that a seven-fold difference in the growth rates of two populations resulted, not from differences in individual fecundity or viability, but from differences in the way immatures interacted in the two populations.

Density-dependent population growth may thus be considered a population-level phenomenon whose response to loss of genetic variance has not been well studied. The genetic differentiation of demes in subdivided populations owing to random genetic drift plays a major role in Wright's "shifting balance theory" of evolution (Wright, 1931; 1978). According to Wright's theory, the most important evolutionary force is selection among more or less randomly differentiated demes. This interdemic selection occurs by the differential dispersion of those demes in which random genetic drift and directional mass selection have established a system of interacting genes of superior fitness. Because selection at any level of biological organization occurs as a result of the interaction of the phenotype and the environment, the success of interdemic selection in producing genetic change depends on the nature of the relationship between the population phenotype and the genetic composition of the population. In this paper, we present the results of a study of the relationship between the theoretically expected amount of genetic variation within populations and the population's mean phenotype as expressed by the rate of population increase.

\section{MATERIALS AND METHODS}

As part of a study of the effects of population structure on the characteristics of Tribolium populations (Wade and McCauley, 1980; McCauley and Wade, 1980), cultures were reared in the following manner. Populations were initiated with a founding propagule ranging in size from 16 to 96 adults depending on the experimental treatment. Initial propagules for all treatments were drawn at random from the common stock population described in Wade (1977). Experiments were conducted at $29^{\circ} \mathrm{C}$ and 70 per 
cent relative humidity using a medium of 95 per cent by weight sifted whole wheat flour fortified with 5 per cent by weight dried brewer's yeast. Founding propagules were permitted to reproduce for 49 days, about three weeks longer than the egg to adult development time under these conditions of husbandry. After 49 days, all living adults were removed and counted. Subsequently, a new propagule consisting of the appropriate number of adults was chosen at random from each newly expanded population and transferred to fresh medium to found the next generation. This process was repeated every 49 days for 12 generations.

Three different propagule sizes, 16,48 , and 96 adults, were studied and there were 15 replicate populations in each treatment. For each of the three propagule sizes, three levels of "island model" migration (Wright, 1931) between populations were also studied. These levels of migration were 0 per cent, 6.25 per cent, and 12.5 per cent of the propagule per generation. For example, in the treatment with 48 adults and 6.25 per cent migration, 45 adults were chosen at random from each of the 15 replicate populations in this treatment immediately following the census. Each group or propagule was used to establish a new population in fresh medium. At this same time three migrant adults $([3 / 48] \times 100=6.25$ per cent $)$ were also added to each of the 15 populations. The total size of the founding propagule is thus 48 adults, 45 adults from one population plus 3 migrants. Each of these migrants was obtained by drawing a population at random and with replacement from among the 15 populations in the treatment and then randomly choosing a single migrant individual from the randomly drawn population. Hence, for this treatment, the total number of random draws of populations at each generation was 45 ( 3 migrants per population $\times 15$ replicate populations), one for each migrant. Because the probability of a migrant coming from one population was the same as the probability of it coming from any other population, this is "island model" migration.

The entire experimental array conformed to a two-factor analysis of variance design; the factors being propagule size and migration rate. There were 9 treatments, each with 15 replicates, for a total of 135 populations ( 3 propagule sizes $\times 3$ migration rates $\times 15$ replicates per treatment $=135$ ).

The purpose of this paper is to describe the temporal changes observed in the mean rate of population increase in each of these 9 population structures and to relate these observations to the theoretically predicted loss of heterozygosity within populations. Although all treatments were reared in the same amount of medium ( $24 \mathrm{~g}$ ), initial densities and, consequently, productivities were affected by the size of the founding propagule (Wade and McCauley, 1980). For this reason, to facilitate treatment comparisons, additional populations were founded from the experimental lines at every second generation. This was done by selecting at random 6 of the 15 replicate populations from each treatment and establishing 4 additional populations from each of these 6 replicates in $8 \mathrm{~g}$ of medium with a uniform propagule size of 16 adults. The numbers of adults in these additional populations after 49 days of population growth are thus comparable over all treatments and observed differences in productivity are the result of the cumulative effects of population structure rather than the demographic and density-dependent properties associated with each propagule size. The total number of additional populations founded was 216 every second generation ( 9 treatments $\times 6$ replicates per treatment $\times 4$ additional 
populations per replicate $=216$ ). It is the productivities of these populations that will be analyzed in relation to the theoretically expected levels of within-population heterozygosity.

\section{RESULTS}

Table 1 represents the grand mean (all replicates of all experimental treatments) number of adults produced at generations $3,5,7,9$, and 11 , by

TABLE 1

The grand mean number of $F_{1}$ adults
produced by 16 parental adults at every
other generation
Generation
3

the additional populations. The entire data set can be analyzed as a mixed model three-factor analysis of variance with the two experimental factors, propagule size and migration rate fixed, and time in generations a random factor. The results of such an analysis are shown in table 2 . The confounding of the generation to generation fluctuations in numbers, illustrated in table 1 , with any long term trends obscures the meaning of the one significant interaction term (migration rate $\times$ time). Of chief interest here are the statistically significant effects of the two experimental factors. Smaller propagule sizes and lower migration rates significantly reduce population growth rates over time (see table 2 ). We hypothesize that this occurs as a consequence of a loss of genetic variation within populations and test this hypothesis by comparing our observations with the theoretical predictions of population genetics.

The proportion of heterozygosity that is expected to be present at neutral loci in a finite population without mutation can be expressed as

$$
H_{t}=H_{0}\left(1-f_{t}\right)
$$

TABLE 2

Three-factor mixed model analysis of variance of population growth rate as measured by the numbers of adults produced by propagules of 16 adults in a 49 day period. The fixed factors are the experimental treatments, propagule size $(A)$ and migration rate $(\mathrm{B})$, and the random factor is generation number $(\mathrm{C})$

\begin{tabular}{crrrc} 
Factor & \multicolumn{1}{c}{ MS } & d.f. & F & $P$ \\
$\mathrm{~A}$ & 25,288 & 2 & 21.61 & $<0.001$ \\
$\mathrm{~B}$ & 28,227 & 2 & $6 \cdot 01$ & $<0 \cdot 010$ \\
$\mathrm{C}$ & 48,804 & 4 & $40 \cdot 40$ & 0.001 \\
$\mathrm{~A} \times \mathrm{B}$ & 1570 & 4 & 1.30 & n.s. \\
$\mathrm{A} \times \mathrm{C}$ & 1170 & 8 & 0.97 & n.s. \\
$\mathrm{B} \times \mathrm{C}$ & 4697 & 8 & 3.89 & $<0.010$ \\
$\mathrm{~A} \times \mathrm{B} \times \mathrm{C}$ & 1447 & 16 & 1.20 & n.s. \\
error & 1208 & 225 & - & -
\end{tabular}


where

$$
f_{t}=\left(\left[1 / 2 N_{e}\right]+\left[1-1 / 2 N_{e}\right] f_{t-1}\right)(1-m)^{2},
$$

$H_{0}$ represents the amount of heterozygosity in the original stock, and $H_{t}$, the amount present after $t$ generations at the effective population size, $N_{e}$. In addition, $f_{t}$ is the genetical correlation between randomly chosen pairs of alleles from the same population in generation $t$, and $m$ is the rate of island model migration between populations (Crow and Kimura, 1970, p. 269).

Assuming random mating within populations and a nearly equal sex ratio, propagule size may be substituted for $N_{e}$ (Wade, 1980), migration rate for $m$, and generation number for $t$ to predict the expected relative loss of heterozygosity with time. One well known consequence of these equations is that large effective population sizes and high rates of migration tend to retard the rate of loss of genetic variance within populations.

These expected relative levels of heterozygosity may be compared to the observed growth rates of the populations. Because generation to generation fluctuations in population size tend to affect the entire experiment, population growth associated with $H_{0}$ will be estimated independently every other generation as the mean growth rate of the $96-12.5$ per cent treatment. This treatment has the largest propagule size (96) and the highest migration rate $(12.5$ per cent) and, on theoretical grounds, would be expected to lose very little genetic variance over the course of the experiment (less than 5 per cent after 11 generations). The $H_{t} / H_{0}$ ratios predicted from the population histories of each treatment through time are presented in table 3. The observed ratios (treatment mean productivity/mean productivity of the $96-12 \cdot 5$ per cent treatment at generation $t$ ) are presented in table 4 . The correlation between the elements in the $5 \times 8$ matrix of table 3 and the associated elements in table 4 indicates the strength of the relationship between the predicted loss of heterozygosity and the observed population growth rates. The product moment correlation of these variables, $r$, is equal to 0.75 (d.f. $=38, P<0.01$ ), demonstrating that such a relationship exists: population growth rates decline as within population heterozygosity is lost. (Although the degrees of freedom at the populational level are 38 , estimating this correlation involved the census of $1.25 \times 10^{6}$ individual beetles.)

This relationship is illustrated in a different way in fig. 1. The data plotted in this figure were drawn from the larger data set to show the decline in population growth rates over as wide a range of predicted $H_{t} / H_{0}$ ratios as possible. This figure presents the observed relative decline in the population growth rate for the 16-0 per cent treatment as a function of the expected decline in $H_{t} / H_{0}$ through time. It also illustrates that a similar relationship obtains across all treatments at the conclusion of the experiment (generation 11). From these two graphs it can be seen that even a relatively small decline in within-population heterozygosity has a measurable effect upon the rate of population increase.

\section{Discussion}

We found a clear and statistically significant relationship between the structure of these populations of $T$. castaneum and their relative rates of population increase. We found that population growth rate declined as the 

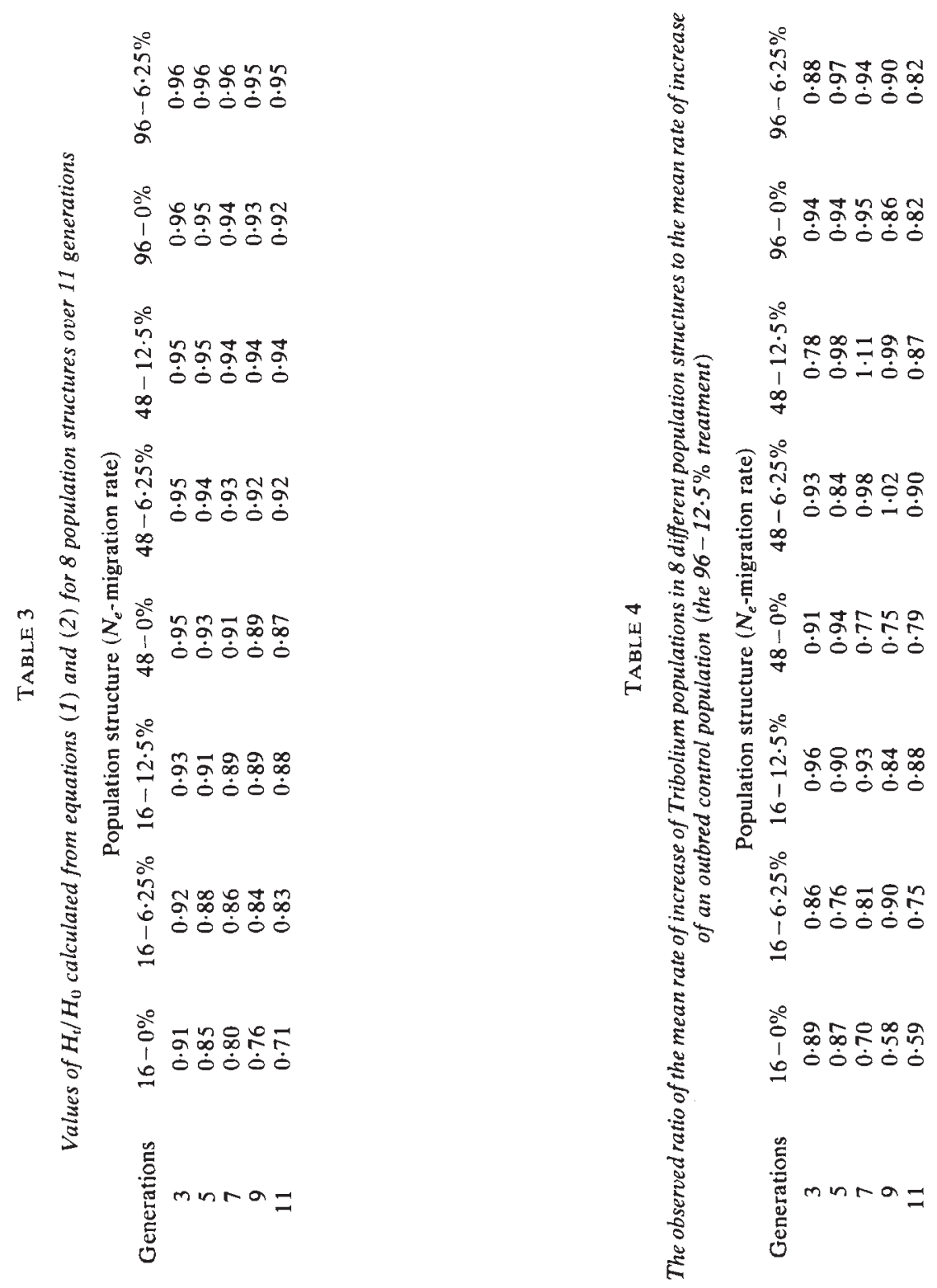


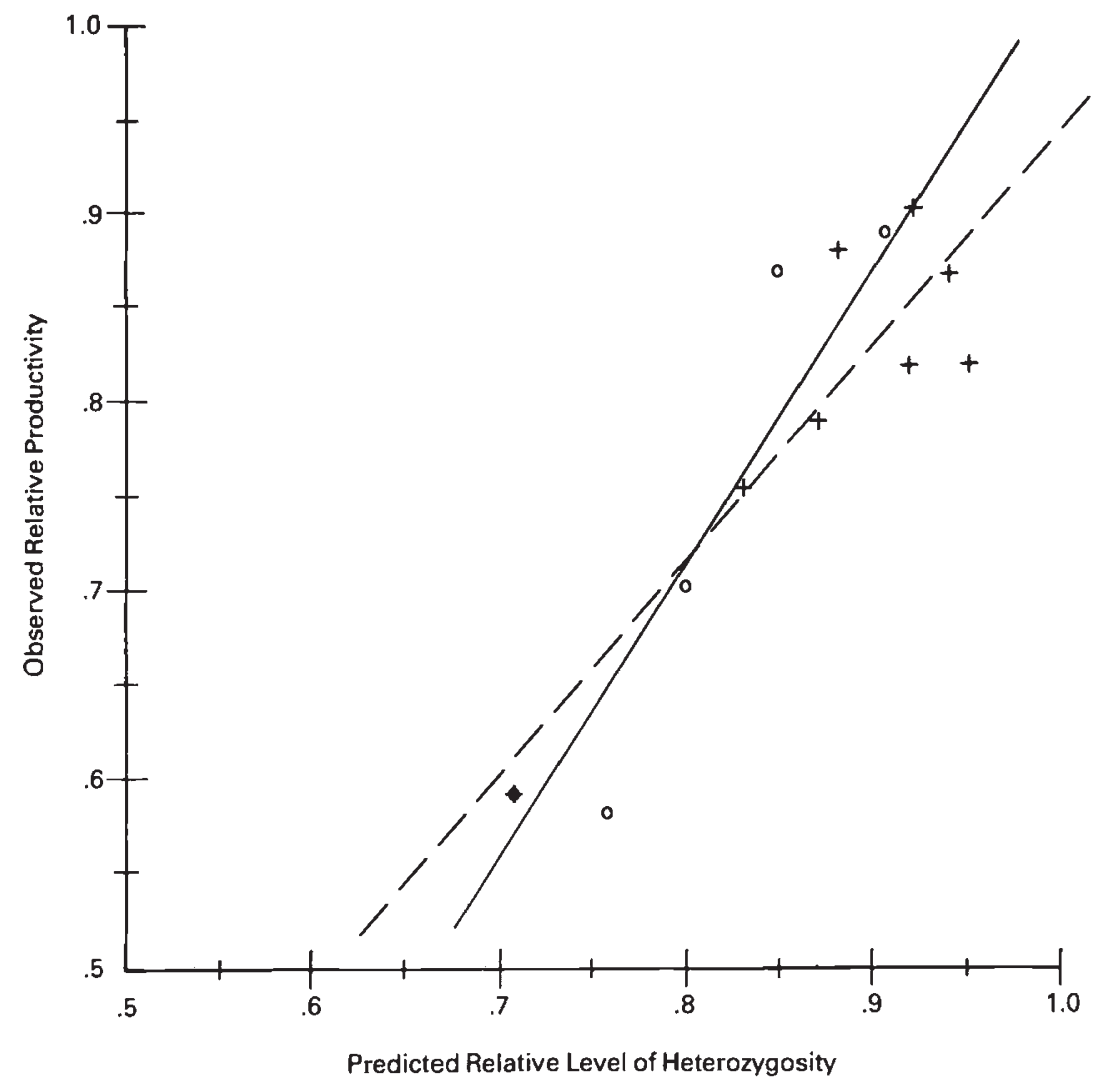

FIG. 1.-Relationship of the predicted ratio of $H_{t} / H_{0}$ as calculated from equations (2) and (3) to the observed relative decline in productivity of adults from the $16-0$ per cent treatment over time (dots with the solid line represent the best least squares fit; $Y=-0 \cdot 72+1 \cdot 80 X$ ). The crosses and the dashed line represent similar information from all eight treatments at generation $11(Y=-0 \cdot 19+1 \cdot 13 X)$.

level of within-population heterozygosity declined. This study demonstrates that the demographic properties of a population may, in part, be determined by the genetical consequences of that population's history. Although no statement regarding the specific traits responsible for these differences in population growth rates can be made at this time, several traits likely to be important to population growth have been shown previously to be sensitive to inbreeding in Tribolium. These include larval viability (Levene et al., 1965; Lopez-Fanjul and Jodar, 1977), and the egg laying rates of fecundated females measured over a two day period (Lopez-Fanjul and Jodar, 1977). In these experiments, however, inbreeding was accomplished by sib mating and the characteristics of individuals were measured at population densities not representative of those experienced by adult and immature beetles in our cultures.

In related papers we have demonstrated the degree to which genetic drift can result in the differentiation of populations with respect to their demographic properties (Wade and McCauley, 1980; McCauley and Wade, 
1980). Specifically, populations with relatively large effective sizes (up to 48 breeding adults) but no migration became significantly differentiated from one another after 14 generations. The present study showed that the population phenotype, growth rate, is very sensitive to genetical changes within populations. An expected loss in heterozygosity as small as 10 per cent resulted in a measurable change in the mean rate of population increase. From table 3 it can be seen that 27 of our 40 measurements were made at $H_{t} / H_{0}$ ratios theoretically expected to be greater than 0.90 . Of these 27 ratios, 25 were observed to be less than 1 (table 4 ). If it were not possible to detect the population effects of a loss of heterozygosity of the order of 10 per cent or less, then we would expect approximately equal numbers of the observed $H_{t} / H_{0}$ ratios to be greater than and less than one. Using a sign test on the observed data, the null hypothesis, that there is no measurable effect on population growth rate when $H_{t} / H_{0}>0.90$, is rejected at the 0.01 level. Clearly, we are detecting the effects of rather mild inbreeding. It is well known that the equilibrium value of $f$ in subdivided populations with island model migration is approximately $(1 /[4 \mathrm{Nm}+1])$. Because a migration rate of one migrant $(m=1 / N)$ per population per generation results in an equilibrium value of $f$ of $0 \cdot 2$, populations exchanging even a small number of migrants are generally considered to be a single panmictic unit. The present study illustrates that populations may indeed be sensitive to $f$ values of the order of 0.1 and implies that genetic drift can play a considerable role in determining the characteristics of populations even when they are exchanging migrants.

It is surprising to us that the observed changes over time in such a complexly determined population phenotype could be fitted so well by theory based on the effects of neutral and additively acting genes. That is, the pattern observed for mean population growth rate is similar to that expected for a simple individual trait subjected to inbreeding depression. One possible explanation concerns our method of standardizing the population performances of all treatments to that of the most outbred treatment (96-12.5 per cent). This may in some way have "factored out" the effects of the interactions between individuals and permitted the expected effects of inbreeding depression on individual traits to be seen. It is also possible that the positive and negative interactions between individuals were partitioned between populations in such a way that the effects of these interactions on the mean productivity of any treatment cancelled one another out. This may have permitted the effects on offspring numbers of the theoretically expected inbreeding depression of individual traits to be measured. Additional studies now in progress may help in deciding which of these possibilities is correct.

Acknowledgements.-We would like to thank both Russ Lande for his constructive comments and Ora Lee Mathews for her technical assistance. Financial support was provided by NIH grant no. $6 \mathrm{M} 22523$ awarded to the junior author.

\section{REFERENCES}

BIRCH, L. C., PARK, T., AND FRANK, M. B. 1951. The effect of intraspecies and interspecies competition on the fecundity of two species of flour beetles. Ecology, 5, 116-132.

CROW, J. F., AND KimurA, M. 1970. An Introduction to Population Genetics Theory. Harper and Row, New York, New York. 
KENCE, A. 1973. The effects of variation in larval development on laboratory populations of Tribolium and houseflies. Ph.D thesis, State University of New York at Stony Brook, New York.

KING, C. E., AND DAwson, P. S. 1972. Population biology and the Tribolium model. Evol. Biol., 5, 133-227.

LEVENE, H., LERNER, I. M., SOKOLOFF, A., HO, F. K., AND FRANKLIN, I. R. 1965. Genetic load in Tribolium. Proc. Natl. Acad. Sci. U.S.A., 53, 1042-1050.

LOPEZ-FANJUL, C., AND JODAR, B. 1977. The genetic properties of egg laying of virgin females of Tribolium castaneum. Heredity, 39, 251-258.

McCAULEY, D. E. 1978. Demographic and genetic responses of two strains of Tribolium castaneum to a novel environment. Evolution, 32, 398-415.

McCAULEY, D. E., AND WADE, M. J. 1980. Group selection: the genetic and demographic basis for the phenotypic differentiation of small populations of Tribolium castaneum. Evolution, 34, 813-821.

MERTZ, D. B. 1972. The Tribolium model and the mathematics of population growth. Annu. Rev. Ecol. Syst., 3, 51-78.

MERTZ, D. B., AND ROBERTSON, J. R. 1970. Some developmental consequences of handling, egg-eating, and population density for flour beetle larvae. Ecology, 51, 989-998.

OSBORNE, J. w. 1968. Inter- and intra-species copulation effect on net fecundity in Tribolium confusum. Trans. Missouri Acad. Sci., 2, 53-57.

PARK, T. 1934. Studies in population physiology III. The effect of conditioned flour upon the productivity and population decline of Tribolium confusum. J. Exp. Zool., 68, 167-182.

PARK, T. 1935. Studies in population physiology IV. Some physiological effects of conditioned flour upon Tribolium confusum Duval and its populations. Physiol. Zool, , 8, 91-115.

PARK, T. 1938a. A note on the size and composition of old Tribolium confusum populations. Amer. Natur., 72, 24-33.

PARK, T. 1938b. Studies in population physiology VIII. The effect of larval population density on the post-embryonic development of the flour beetle, Tribolium confusum Duval. $J$. Exp. Zool., 79, 51-70.

PARK, T., AND WOOLLCOTT, N. 1937. Studies in population physiology VIII. The relation of environmental conditioning to the decline of Tribolium confusum populations. Physiol. Zool. , 10, 197-211.

SOKOLOFF, A. 1974. The Biology of Tribolium Vol. 2. Oxford University Press, London.

SOKOLOFF, A. 1977. The Biology of Tribolium Vol. 3. Oxford University Press, London.

SONLEITNER, F. J. 1961. Factors affecting egg cannibalism and fecundity in populations of adult Tribolium castaneum Herbst. Physiol. Zool., 34, 233-255.

WADE, M. J. 1977. An experimental study of group selection. Evolution, 31, 134-153.

WADE, M. J. 1979. The primary characteristics of Tribolium populations group selected for increased and decreased population size. Evolution, 33, 749-764.

WADE, M. J. 1980. Effective population size: the effects of sex, genotype, and density on the mean and variance of offspring numbers in the flour beetle, Tribolium castaneum. Genet. Res., 36, 1-10.

WADE, M. J., AND McCAULEY, D. E. 1980. Group selection: the phenotypic and genotypic differentiation of small populations. Evolution, 34, 799-812.

WRIGHT, S. 1931. Evolution in Mendelian populations. Genetics, 16, 93-159.

WRIGHT, S. 1977. Evolution and the Genetics of Populations: Vol. III. University of Chicago Press, Chicago, Illinois.

WRIGHT, S. 1978. Evolution and the Genetics of Populations: Vol. IV. University of Chicago Press, Chicago, Illinois. 